\begin{tabular}{|c|l|}
\hline Title & Ferromagnetic Ising spin systems on the growing random tree \\
\hline Author(s) & Hasegawa, Takehisa; Nemoto, Koji \\
\hline Citation & $\begin{array}{l}\text { Physical review E, 80(2), 026126 } \\
\text { https://doi.org/_0.1103/PhysRevE.80.026126 }\end{array}$ \\
\hline Issue Date & 2009-08 \\
\hline Doc URL & http://hdl.handle.net/2115/39337 \\
\hline Rights & O2009 The A merican Physical Society \\
\hline Type & article \\
\hline File Information & PRE80-2_026126.pdf \\
\hline
\end{tabular}

Instructions for use 


\title{
Ferromagnetic Ising spin systems on the growing random tree
}

\author{
Takehisa Hasegawa ${ }^{*, \dagger}$ and Koji Nemoto \\ Department of Physics, Hokkaido University, Kita 10, Nishi 8, Kita-Ku, Sapporo, Hokkaido 060-0810, Japan
}

(Received 19 April 2008; revised manuscript received 23 March 2009; published 26 August 2009)

\begin{abstract}
We analyze the ferromagnetic Ising model on a scale-free tree; the growing random tree model with the linear attachment kernel $A_{k}=k+\alpha$. We derive an estimate of the divergent temperature $T_{s}$ below which the zero-field susceptibility of the system diverges. Our result shows that $T_{s}$ is related to $\alpha$ as $\tanh \left(J / T_{s}\right)$ $=\alpha /[2(\alpha+1)]$, where $J$ is the ferromagnetic interaction. An analysis of exactly solvable limit for the model and numerical calculation supports the validity of this estimate.
\end{abstract}

DOI: 10.1103/PhysRevE.80.026126

PACS number(s): 89.75.Fb, 05.50.+q, 89.75.Hc

\section{INTRODUCTION}

Many real-world systems range from the structure of Internet or www to social relationship between human society or prey-predator relationship in food webs are described topologically as scale-free networks (SFNs) [1-3]. In a SFN, the degree distribution $P(k)$, where degree $k$ is the number of edges connected to a node, has a power-law decay $P(k)$ $\propto k^{-\gamma}$. The degree exponent $\gamma$ takes $2<\gamma<3$ in many real networks [4]. SFN studies have been carried out actively in recent years, including various processes taking thereon, e.g., network failure, spread of infections, or interacting systems, which attract numerous applications and fundamental interests about critical phenomena $[2,3,5]$. Among them, the ferromagnetic Ising model on SFNs is a basic prototype to understand how network topology influences cooperative behaviors on SFNs. Indeed, previous studies have demonstrated the extreme influence of the network topology [6-15]. For example, Dorogovtsev et al. [9] analyzed the Ising model on uncorrelated SFNs by the Bethe approach to show that the phase transition exists at a finite temperature for $\gamma>3$, while the system remains in the ferromagnetic phase at any finite temperature for $\gamma \leq 3$, and its critical exponents vary depending on $\gamma$.

While various systems on uncorrelated SFNs, in which the Bethe approach is very effective, have been studied to show simple and interesting pictures, many works have reported that the systems on some growing networks show quite different phase transitions from those on uncorrelated SFNs $[5,11,12,16]$. The effects of growth mechanisms on phase transitions are still unclear. In this paper, we analyze the Ising model on a simple growing tree model to investigate this problem. It is well known that the ferromagnetic Ising model on trees has no spontaneous magnetization at any finite temperature in contrast to that on regular lattices, while its zero-field susceptibility remains to diverge below a certain temperature $T_{s}$ (we call it the divergent temperature) [17-24]. In other words, the system is extremely sensitive to the external field below $T_{s}$. How does the so-called preferen-

\footnotetext{
*Present address: Graduate School of Information Science and Technology, The University of Tokyo, 7-3-1, Hongo, Bunkyo-ku, Tokyo, 113-8656, Japan.

†hase@statphys.sci.hokudai.ac.jp
}

tial attachment in a growth process influence the sensitivity of a resulting tree to the external field? The aim of this paper is to clarify the relation between the divergent temperature $T_{s}$ and the degree distribution $P(k)$.

In this paper, we use the growing random tree (GR tree) (the Dorogovtsev-Mendes-Samukhin model [25]) introduced by Krapivsky and co-workers [26]. The GR tree is probabilistically generated as a sampled tree $T_{N}$ with $N$ nodes as follows. One starts with one isolated node. At each time step, a new node is added and links to one of pre-existing nodes. The probability that the new node links to a node with degree $k$ is proportional to the attachment kernel $A_{k}$. In this paper, we focus on the linear attachment kernel $A_{k}=k+\alpha(\alpha>-1)$. The degree distribution of resulting trees depends on offset $\alpha$ in the attachment kernel. For the infinite offset $\alpha \rightarrow \infty$, the degree distribution reduces to the exponential form $P(k)$ $=2^{-k}$. For finite offset $\alpha$, the degree distribution satisfies a power-law decay $P(k) \propto k^{-\gamma}$, where the degree exponent is $\gamma=3+\alpha$ [26]. Particularly, the GR tree with no offset $\alpha=0$ corresponds to the so-called Barabási-Albert tree [27-30], which has the degree exponent $\gamma=3$. We investigate how the divergent temperature $T_{s}$ is related to $\alpha$. Our results show that, like the transition temperature of the Ising model on uncorrelated SFNs, $T_{s}$ on the GR tree is larger as the degree exponent $\gamma$ is smaller and reaches the infinity for $\gamma \leq 3$.

This paper is organized as follows. In Sec. II, we introduce the Ising model on the GR tree. In Sec. III, we derive the exact expression for the system susceptibility and the divergent temperature for the infinite offset case $\alpha \rightarrow \infty$. In Sec. IV, we give an estimate of $T_{s}$ for finite offset cases. In Sec. V, we show our numerical results to support the validity of our estimate and suggest that for no offset case, $T_{s}$ diverges and an unusual scaling exists. Section VI is devoted to the summary.

\section{MODEL}

In this section, we introduce our model: the Ising model on the GR tree. The Hamiltonian is

$$
\mathcal{H}=-J \sum_{\langle i j\rangle} S_{i} S_{j}-h \sum_{i} S_{i}
$$

where $J(>0)$ is the ferromagnetic interaction, $h$ is the external magnetic field, and $S_{i}(= \pm 1)$ is the Ising spin variable on the node $i$. The first sum is over all edges, and the second one 
is over all nodes. In the following sections, we focus on the zero-field susceptibility of this model. The zero-field susceptibility is expressed in terms of the spin-spin correlation functions as $\chi_{\text {sys }}=\frac{1}{N} \sum_{i, j=1}^{N}\left\langle S_{i} S_{j}\right\rangle$, where the angular bracket denotes the usual thermal average. For trees, the correlation function between two Ising spins $S_{i}$ and $S_{j}$ on a sampled tree $T_{N}$ is given as [21]

$$
\left\langle S_{i} S_{j}\right\rangle=t^{d_{i, j}\left(T_{N}\right)},
$$

where $t=\tanh (\beta J), \beta=1 / T, T$ being the temperature, and $d_{i, j}\left(T_{N}\right)$ is the path length between the nodes $i$ and $j$ on the tree $T_{N}$. Accordingly, the one-spin susceptibility $\chi_{i}\left(T_{N}\right)$ of a spin on the node $i$ of a sampled tree $T_{N}$ is

$$
\chi_{i}\left(T_{N}\right)=\beta \sum_{j=1}^{N} t^{d_{i, j}\left(T_{N}\right)},
$$

and the system susceptibility

$$
\chi_{\text {sys }}\left(T_{N}\right)=\frac{1}{N} \sum_{i=1}^{N} \chi_{i}\left(T_{N}\right)=\frac{\beta}{N} \sum_{i, j=1}^{N} t^{d_{i, j}\left(T_{N}\right)} .
$$

Note that the system susceptibility is related with the socalled average correlation volume $\xi_{V}[5]: \xi_{V}=T \chi_{\text {sys }}$.

\section{INFINITE OFFSET CASE}

In this section, we consider the GR tree with the infinite offset $\alpha \rightarrow \infty$. We derive the exact form for the mean system susceptibility $\overline{\chi_{\text {sys }}}(N, T)$ and $T_{s}$. Here $\bar{A}=\Sigma_{T_{N}} P\left(T_{N}\right) A\left(T_{N}\right)$, and $P\left(T_{N}\right)$ is the normalized probability of a tree $T_{N}$. Suppose that $T_{N+1}$ is created by attaching the $(N+1)$ th node to the $n$th node of a pre-existing tree $T_{N}$. Then the distance from the new node to all others is given as $d_{i, N+1}\left(T_{N+1}\right)$ $=d_{N+1, i}\left(T_{N+1}\right)=1+d_{n, i}\left(T_{N}\right)$ for $1 \leq i \leq N$ [30]. The diagonal element is zero: $d_{N+1, N+1}\left(T_{N+1}\right)=0$. Note that for trees, a path between each pair of nodes is unique, so the matrix elements do not change their values once formed during a growth process. So we obtain the following recursion relation for the averaged total susceptibility $\beta V_{N}=N \chi_{\text {sys }}(N, T)$ :

$$
\begin{aligned}
V_{N+1} & =\sum_{T_{N+1}} P\left(T_{N+1}\right) \sum_{i, j=1}^{N+1} t^{d_{i, j}\left(T_{N+1}\right)} \\
= & \sum_{T_{N+1}} P\left(T_{N+1}\right) \sum_{i, j=1}^{N} t^{d_{i, j}\left(T_{N+1}\right)} \\
& +2 \sum_{T_{N+1}} P\left(T_{N+1}\right) \sum_{i=1}^{N} t^{d_{i, N+1}\left(T_{N+1}\right)}+1 .
\end{aligned}
$$

The first term of the right-hand side is

$$
\sum_{T_{N+1}} P\left(T_{N+1}\right) \sum_{i, j=1}^{N} t^{d_{i, j}\left(T_{N+1}\right)}=\sum_{T_{N}} P\left(T_{N}\right) \sum_{i, j=1}^{N} t^{d_{i, j}}\left(T_{N}\right)=V_{N},
$$

and the second term is

$$
\begin{aligned}
& \sum_{T_{N+1}} P\left(T_{N+1}\right) \sum_{i=1}^{N} t^{d_{i, N+1}\left(T_{N+1}\right)} \\
& \quad=\sum_{T_{N}} P\left(T_{N}\right) \sum_{n=1}^{N} P\left(n \mid T_{N}\right) \sum_{i=1}^{N} t^{1+d_{i, n}\left(T_{N}\right)} .
\end{aligned}
$$

Here we use $P\left(T_{N+1}\right)=P\left(T_{N}\right) P\left(n \mid T_{N}\right)$, where $P\left(n \mid T_{N}\right)$ is the conditional probability that the newly added node links to a pre-existing node labeled as $n$ on the tree $T_{N}$. Combining Eqs. (6) and (7) with Eq. (5) gives the evolution of $V_{N}$ as

$$
V_{N+1}=V_{N}+1+2 t \sum_{T_{N}} P\left(T_{N}\right) \sum_{n=1}^{N} P\left(n \mid T_{N}\right) \sum_{i=1}^{N} t^{d_{i, n}\left(T_{N}\right)} .
$$

Although it is hard to solve $V_{N}$ explicitly for finite offset cases since the probability $P\left(n \mid T_{N}\right)$ is proportional to the kernel $A_{k}$, the infinite offset case is within reaching distance. In this case, the conditional probability is independent of which node is attached: $P\left(n \mid T_{N}\right)$ for any $n$. Thus, Eq. (8) is evaluated as

$$
V_{N+1}=V_{N}+1+\frac{2 t}{N} \sum_{T_{N}} P\left(T_{N}\right) \sum_{n=1}^{N} \sum_{i=1}^{N} t^{d_{i, n}\left(T_{N}\right)}=1+\left(1+\frac{2 t}{N}\right) V_{N} .
$$

This recursion equation is solved explicitly to obtain the mean system susceptibility as

$$
\frac{\overline{\chi_{\text {sys }}}(N, T)}{\beta}=\frac{V_{N}}{N}=1+t+2 t \sum_{m=2}^{N-1} \frac{1}{m(m+1)} \prod_{k=1}^{m-1}\left(1+\frac{2 t}{k}\right) .
$$

By evaluating the temperature below which the system susceptibility (10) diverges, we find that the divergent temperature is given as (see Appendix A)

$$
\tanh \left(J / T_{s}\right)=\frac{1}{2}
$$

Moreover, expanding Eq. (10) around the divergent temperature, we obtain the finite-size scaling form for the infinite offset case,

$$
\overline{\chi_{\text {sys }}}(N, T) \simeq(\ln N) f\left[\left(T-T_{s}\right) \ln N\right],
$$

where $f(x)$ is a scaling function in this case.

\section{FINITE OFFSET CASE}

In this section, we proceed to finite offset cases. We give an estimate of $T_{s}$ by calculating a lower bound of the system susceptibility. First, we can calculate approximately the onespin susceptibility of the initial node $\overline{\chi_{1}}$. Let us assign a level to each node of the GR tree according to the distance $l$ from the initial node. The initial node is placed at the zeroth level, and a new node which links to a node at the $l$ th level is placed at the $(l+1)$ th level [27]. Let $n_{N}^{(l)}$ denote the mean number of nodes at the $l$ th level on trees with $N$ nodes. Then the total degree of nodes at the $l$ th level is equal to $n_{N}^{(l)}$ 
$+n_{N}^{(l+1)}$. When the new node is added to a tree with $N$ nodes, the probability that the new node links to any node at the $l$ th level is approximately $\left(n_{N}^{(l)}+n_{N}^{(l+1)}+\alpha n_{N}^{(l)}\right) /[(2+\alpha) N-1]$. Here, this new node is stationed at the $(l+1)$ th level, so we obtain

$$
n_{N+1}^{(l+1)}=n_{N}^{(l+1)}+\frac{c_{1} n_{N}^{(l)}+n_{N}^{(l+1)}}{c_{2} N-1} \quad(l \geq 1),
$$

where $c_{1}=1+\alpha, c_{2}=2+\alpha$, and the initial condition is $n_{N}^{(0)}$ $=1$ for all $N$. Now, we introduce the generating function

$$
G_{N}(t)=\sum_{l=0}^{\infty} n_{N}^{(l)} t^{l}
$$

Note that $G_{N}(t)$ is related to $\overline{\chi_{1}}$ as $\overline{\chi_{1}}=\beta G_{N}(t)$. From Eq. (13), we obtain the recursion relation for the generating function as follows:

$$
\left(c_{2} N-1\right) G_{N+1}(t)=\left(c_{2} N+c_{1} t\right) G_{N}(t)-1 .
$$

It is easily solved that

$$
G_{N}=1+t \frac{\Gamma\left(2-c_{2}^{-1}\right)}{\Gamma\left(1+c_{2}^{-1} c_{1} t\right)} \sum_{M=1}^{N-1} \frac{\Gamma\left(M+c_{2}^{-1} c_{1} t\right)}{\Gamma\left(M+1-c_{2}^{-1}\right)} .
$$

For $N \gg 1$, the summation of the second term can be approximated as

$$
\sum_{M=1}^{N-1} \frac{\Gamma\left(M+c_{2}^{-1} c_{1} t\right)}{\Gamma\left(M+1-c_{2}^{-1}\right)} \simeq \sum_{M=1}^{N-1} M^{\left(c_{1} t+1 / c_{2}\right)-1} \simeq N^{\left(c_{1} t+1\right) /\left(c_{2}\right)},
$$

so that

$$
G_{N}(t) \simeq N^{\left(c_{1} t+1\right) /\left(c_{2}\right)}
$$

Thus, we obtain the one-spin susceptibility of the initial node as

$$
\overline{\chi_{1}}(N, T)=\beta G_{N}(t) \simeq \beta N^{[1+(1+\alpha) t] /(2+\alpha)},
$$

which diverges for any $T$ and any $\alpha(>-1)$.

Now, we evaluate a lower bound of the system susceptibility. We consider a subtree which consists of a node at the $s$ th level and its descendants. Assuming that the numbers of offspring of the nodes at the same level are all the same, the number of nodes at the $(s+l)$ th level in the subtree is given as $n_{N}^{(s+l)} / n_{N}^{(s)}$. Among the total susceptibility of the subtree, the contribution from the node pairs whose paths go through the level $s$ is $G_{N, s}^{2}-\left[n_{N}^{(s+l)} / n_{N}^{(s)}\right] t^{2} G_{N, s+1}^{2}$, where

$$
G_{N, s}=\sum_{l=0}^{\infty} \frac{n_{N}^{(s+l)}}{n_{N}^{(s)}} t^{l}
$$

corresponds to the one-spin susceptibility of the node at the $s$ th level. The total susceptibility of the whole tree is evaluated as

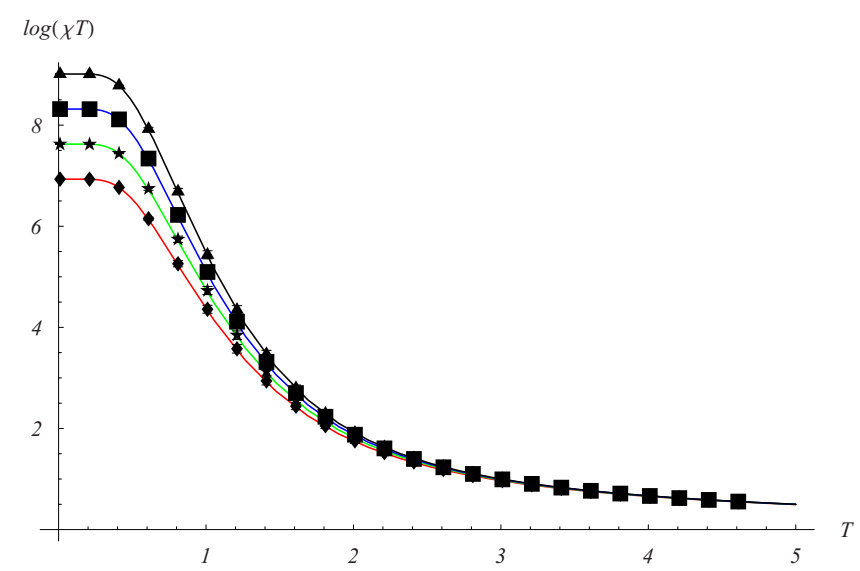

FIG. 1. (Color online) The system susceptibility for the infinite offset by the analytical results [Eq. (10)] (lines) and the numerical ones (points). The numbers of nodes are taken $N=2^{10}$ (red), $2^{11}$ (green), $2^{12}$ (blue), and $2^{13}$ (black), from bottom to top. The average is taken over 100 samples.

$$
\begin{aligned}
N T \overline{\chi_{\mathrm{sys}}}(N, T) & =\sum_{s=0}^{\infty}\left(G_{N, s}^{2}-t^{2} G_{N, s+1}^{2} \frac{n_{N}^{(s+l)}}{n_{N}^{(s)}}\right) n_{N}^{(s)} \\
& =n_{N}^{(0)} G_{N, 0}^{2}+\left(1-t^{2}\right) \sum_{s=0}^{\infty} n_{N}^{(s+l)} G_{N, s+1}^{2} .
\end{aligned}
$$

The second term is non-negative for $0 \leq t \leq 1$, so we obtain a lower bound of the system susceptibility as

$$
T \overline{\chi_{\mathrm{sys}}}(N, T) \geq \frac{1}{N} n_{N}^{(0)} G_{N, 0}^{2}=\frac{1}{N} G_{N}^{2} \simeq N^{\left\{2\left[\left(1+c_{1} t\right) /\left(c_{2}\right)-1\right]\right\}} .
$$

Note that the exponent includes $t$. By evaluating where this bound diverges, we obtain an estimate of $T_{s}$,

$$
\tanh \left(J / T_{s}\right)=\frac{\alpha}{2(\alpha+1)},
$$

which reduces the exact solution (11) for the infinite offset $\alpha \rightarrow \infty$. This relation indicates that as the offset is smaller, the divergent temperature is higher. Particularly, we immediately find that $T_{s}$ diverges for $-1<\alpha \leq 0$.

\section{NUMERICAL CALCULATIONS}

In this section, we calculate the zero-field susceptibilities numerically for the GR trees with several values of offset $\alpha$. We generate trees for a given offset and calculate their susceptibilities by using Eq. (2). First, we show the results for the infinite offset case $\alpha \rightarrow \infty$. Figure 1 compares the numerical result for the system susceptibility with the analytical forms [Eq. (10)]. For convenience, we set $J=1$. We find that the analytical forms agree well with the numerical ones. Figure 2 plots the finite-size scaling (12) around the divergent temperature. The number of nodes is taken from $2^{10}$ to $2^{13}$. The system susceptibilities are averaged over 100 samples. We find that the scaling works quite well. 


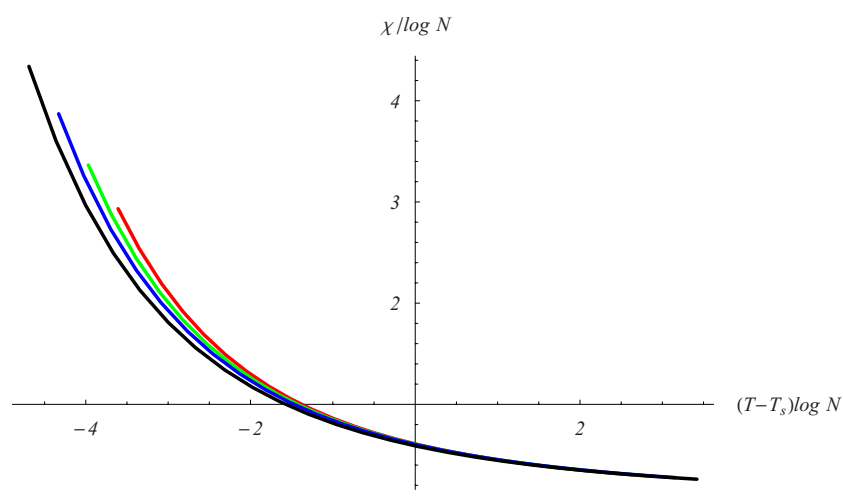

FIG. 2. (Color online) Finite-size scaling (12) of the system susceptibility around $T_{s}$ for the infinite offset. The numbers of nodes are taken $N=2^{10}$ (red), $2^{11}$ (green), $2^{12}$ (blue), and $2^{13}$ (black), from top to bottom. The average is taken over 100 samples.

Next, we turn to finite offset cases. We assume that a finite-size scaling form for finite offset cases follows that for the infinite offset case (12). Figures 3 plots the finite-size scaling around our estimate $T_{s}$ for the mean system susceptibility with the offsets 6,4 , and 1 . As a result, we find that our finite-size scaling similar to that for the infinite offset (12) is quite well fitted as long as an offset is not small. These results support that our estimate gives the exact divergent temperature. On the other hand, our scaling does not work well for the small offsets, e.g., $\alpha=1$ or 2, where scaling exponents there may depend on the offset strongly.

Finally, we consider no offset case. In Fig. 4(a), we plot the mean system susceptibilities $\ln \left[\overline{\chi_{\text {sys }}}(N, T)\right]$ with several nodes from $N=2^{10}$ to $2^{15}$. Now we rescale these susceptibilities as $\ln \left[T \bar{\chi}_{\text {sys }}(N, T)\right] / \ln N$. The rescaled system susceptibilities are quite well fitted for very wide temperature range as seen in Fig. 4(b). This indicates that $T \overline{\chi_{\text {sys }}}(N, T)$ goes to the infinity as $N \rightarrow \infty$. In addition, our result means that the following unusual scaling for the system susceptibility (in other words, for the average correlation volume $\bar{\xi}_{V}$ ) is satisfied:

$$
T \chi_{\text {sys }}(N, T)=[g(T)]^{\ln N},
$$

where $g(x)$ is a scaling function. This relation is derived partially by the following approximation. The expected number $E_{l}$ of shortest paths of length $l$ for the Barabási-Albert tree with $N$ nodes is given as

$$
E_{l} \sim \frac{N^{2}}{2} \frac{1}{\sqrt{2 \pi \ln N}} e^{-\left[(l-\ln N)^{2} /(2 \ln N)\right]},
$$

for $N \gg 1$ [28]. The system susceptibility is approximately evaluated as

$$
\begin{aligned}
T \overline{\chi_{\text {sys }}}(N, T) & =\frac{1}{N} \sum_{l=0}^{N-1} E_{l} t^{l} \\
& \sim \frac{N}{2} \frac{1}{\sqrt{2 \pi \ln N}} \int_{0}^{N-1} e^{-\left[(l-\ln N)^{2} /(2 \ln N)\right]} t^{l} d l \\
& \sim \frac{1}{2} e^{\ln N\left[1+\ln t+(1 / 2)(\ln t)^{2}\right]}
\end{aligned}
$$
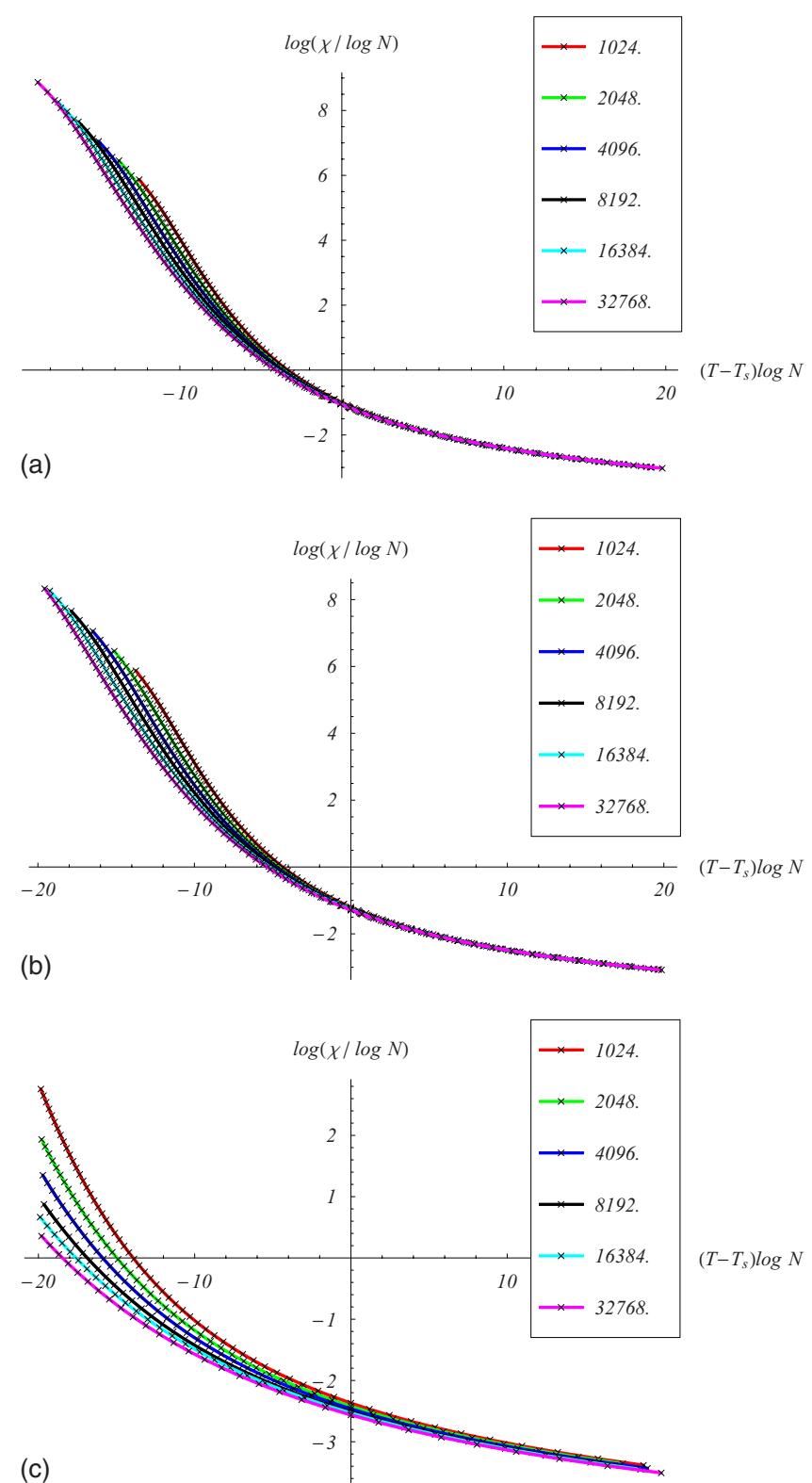

FIG. 3. (Color online) Finite-size scaling of the system susceptibility around our estimate $T_{s}$ [Eq. (23)] for offset $\alpha=6$ (top), 4 (center), and 1 (bottom). The number of nodes is taken from $N$ $=2^{10}$ (top) to $2^{15}$ (bottom). The average is taken over 100 samples.

for $\ln t>-1$. This approximation shows that the system susceptibility $T \chi_{\text {sys }}(N, T)$ holds the scaling relation (24) at least in a low-temperature region. Interestingly, this scaling form remains to be satisfied even at a high temperature where this approximation (26) is not valid.

\section{SUMMARY}

In this paper, we investigated the zero-field susceptibility of the Ising model on the GR tree with the attachment kernel $A_{k}=k+\alpha$. Our main finding of this paper is that the divergent temperature $T_{s}$ of the GR tree with offset $\alpha$ is given by $\tanh \left(J / T_{s}\right)=\alpha / 2(\alpha+1)$ (Fig. 5). The exact expression of the susceptibility for the infinite offset and the finite-size scaling 
(a)

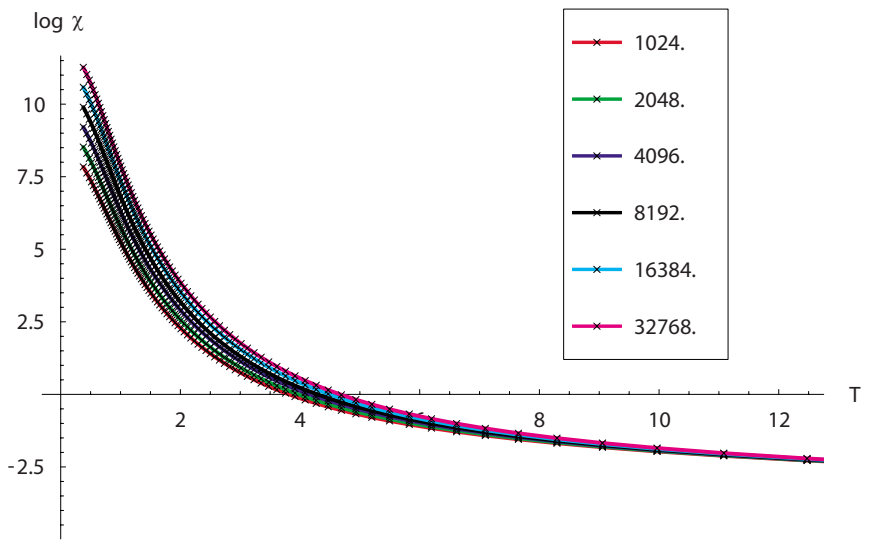

(b)

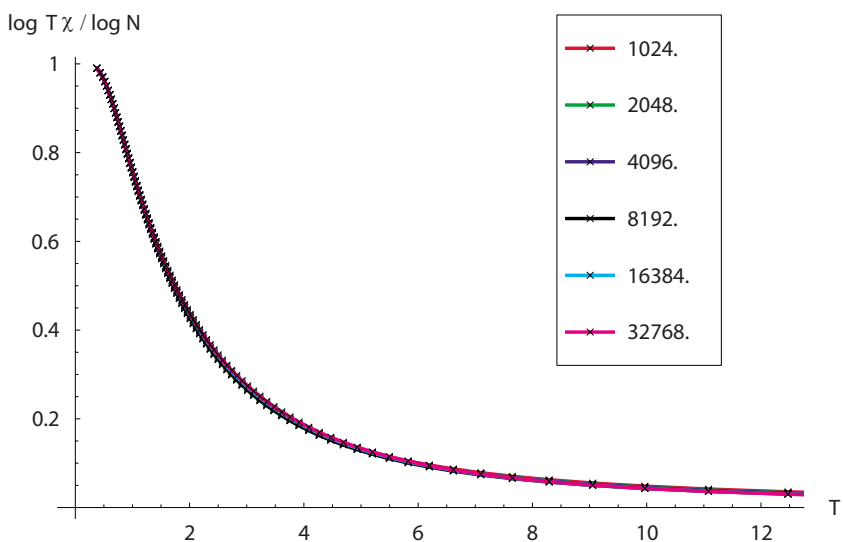

FIG. 4. (Color online) (a) The system susceptibility $\ln \overline{\chi_{\text {sys }}}$ and (b) $\ln \left(T \overline{\chi_{\text {sys }}}\right) / \ln N$ on the GR tree for no offset $\alpha=0$. The number of nodes is taken from $N=2^{10}$ to $2^{15}$. The results are averaged over 100 samples.

of the susceptibilities for finite offsets support that our estimate is exact. The finite-size scaling form (12) implies that $\ln N$ can be regarded as the characteristic system length $L$, so that Eq. (12) can be read as $\overline{\chi_{\text {sys }}} \sim L^{\gamma} f\left(\Delta_{T} L^{1 / \nu}\right)$ with $\gamma=1, \nu$ $=1$.

It is worthwhile to mention the relation between $T_{s}$ and underlying network's structure. From Appendix B, the excess degree $k_{\mathrm{ex}} \equiv\left(\left\langle k^{2}-k\right\rangle\right) /\langle k\rangle$ of the GR tree is given by $k_{\mathrm{ex}}$ $=2(\alpha+1) / \alpha$, so that $\tanh \left(J / T_{s}\right)=1 / k_{\text {ex }}$. Interestingly, a similar relation holds for the transition temperature $T_{c}$ of uncorrelated SFNs, below which the spontaneous magnetization appears, $\tanh \left(J / T_{c}\right)=1 / k_{\text {ex }}[9]$. The GR tree has no ordered phase, but just its susceptibility diverges. The present situation may be similar to the Ising model on the regular Cayley tree. The Ising model on the Cayley tree undergoes a sequence of transitions [18]. These transitions are only seen in the field dependence of the free energy and the $m$ th and higher derivatives of the free energy with respect to the field diverge at the critical temperature $T_{m}$. The sequence interpolates between a first-order transition at $T_{1}=0$ and an infinite order transition at the Bethe-Peierls transition temperature $T_{\infty}=T_{\mathrm{BP}}\left(0=T_{1}<T_{2}<T_{3}<\cdots<T_{\infty}=T_{\mathrm{BP}}\right)$. Above $T=T_{\infty}$, all derivatives are finite. The divergent temperature $T_{s}$ in this work corresponds to $T_{2}$. Where is the transition point $T_{\infty}$ of

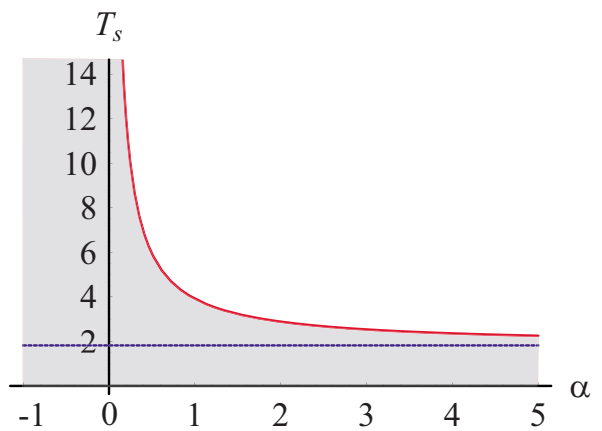

FIG. 5. (Color online) The relation between the divergent temperature $T_{s}$ and offset $\alpha$ of the Ising model on the GR tree. The red-solid line denotes the relation (23) between $\alpha$ and $T_{s}$. The bluedot line denotes $T_{s}$ for the infinite offset $\alpha$. In the blue-colored region, the system susceptibility diverges. the GR tree if a similar sequence exists? The susceptibility (19) of the initial node diverges irrespective of $T$ and $\alpha$, so sufficiently high-field derivatives of the free energy should diverge and, thus, $T_{\infty}$ reaches the infinity. We think that this is also true on any GR tree and the growth mechanism takes $T_{\infty}$ to the infinity.

Finally, we point out that even with same degree distribution, not all trees may behave like the above scenario. The GR tree belongs to growing random trees with the infinite Hausdorff dimension and the small-world property [31]. But there also exist random connected trees which can be scale free, while having the finite Hausdorff dimension and not small-world property in the statistical ensembles of maximum entropy trees (equilibrium random connected trees) [32]. The latter has quite different geometry from the former. Maximum entropy trees favor to generate many long branches and less compact than growing random trees. It would be interesting to examine how spins on such trees behave.

\section{ACKNOWLEDGMENT}

This work is supported by the 21 st Century Center of Excellence (COE) program entitled "Topological Science and Technology," Hokkaido University.

\section{APPENDIX A: THE DERIVATION OF THE DIVERGENT TEMPERATURE OF THE GR TREE WITH THE INFINITE OFFSET}

In this appendix, we derive the divergent temperature of the GR tree with the infinite offset $\alpha \rightarrow \infty$. We rewrite the system susceptibility (10) as

$$
v_{N}=1+t+2 t \sum_{m=2}^{N-1} \frac{1}{m(m+1)} Q_{m},
$$

where 


$$
Q_{m}=\prod_{k=1}^{m-1}\left(1+\frac{2 t}{k}\right) .
$$

One immediately finds that the divergence occurs at $t \geq 1 / 2$ as follows. At $t=1 / 2$, one finds

$$
Q_{m}=\prod_{k=1}^{m-1} \frac{k+1}{k}=m
$$

to reduce the system susceptibility (A1) to

$$
v_{N}=1+t+2 t \sum_{m=2}^{N-1} \frac{1}{m+1}=\sum_{m=1}^{N} \frac{1}{m}
$$

and the system susceptibility diverges in the limit $N \rightarrow \infty$. Since $v_{N}$ increases monotonously with $t$ for any $N$, the system susceptibility diverges at least for $t \geq 1 / 2$. Now we show that the system susceptibility cannot diverge for $t<1 / 2$. From Eq. (A2), we obtain the following relations for $Q_{m}$ :

$$
\sum_{m=2}^{N-1} \frac{1}{m(m+1)} Q_{m}=\sum_{m=2}^{N-1}\left(\frac{1}{m}-\frac{1}{m+1}\right) Q_{m},
$$

and

$$
Q_{m+1}-Q_{m}=\frac{2 t}{m} Q_{m}
$$

The iterative substitutions of Eqs. (A5) and (A6) allow one to the following transformation:

$$
\begin{aligned}
\sum_{m=2}^{N-1} \frac{1}{m(m+1)} Q_{m}= & \frac{1}{2} Q_{2}+\sum_{m=2}^{N-2} \frac{2 t}{m(m+1)} Q_{m}-\frac{1}{N} Q_{N-1} \\
= & \frac{1+2 t}{2} Q_{2}+\sum_{m=2}^{N-3} \frac{(2 t)^{2}}{m(m+1)} Q_{m} \\
& -\frac{2 t}{N-1} Q_{N-2}-\frac{1}{N} Q_{N-1} \\
= & \cdots=\frac{1}{2} \sum_{m=2}^{N-2}(2 t)^{m-1}-\sum_{m=2}^{N-1} \frac{(2 t)^{N-m-1}}{m+1} Q_{m} .
\end{aligned}
$$

In the end, one finds

$$
\begin{aligned}
v_{N} & =1+t+\frac{1}{2} \sum_{m=1}^{N-2}(2 t)^{m-1}-\sum_{m=2}^{N-1} \frac{(2 t)^{N-1-m}}{m+1} Q_{m} \\
& \leq 1+t+\frac{1}{2} \sum_{m=1}^{N-2}(2 t)^{m-1} .
\end{aligned}
$$

This upper bound converges for $t<1 / 2$, so $v_{N}$ does not diverge there. Thus, we find that the divergent temperature $T_{S}$ is decided by $\tanh \left(J / T_{s}\right)=1 / 2$.

\section{APPENDIX B: DERIVATION OF THE EXCESS DEGREE OF THE GR TREE}

In this appendix, we derive the excess degree $k_{\mathrm{ex}} \equiv\left\langle k^{2}\right.$ $-k\rangle /\langle k\rangle$ of the GR tree. $N_{k}(N)$ is defined as the average number of nodes with degree $k$ for trees with $N$ nodes. In network growths, the rate equation for $N_{k}(N)$ is as follows:

$$
\begin{aligned}
N_{k}(N+1)= & N_{k}(N)-\frac{k+\alpha}{(2+\alpha) N-1} N_{k}(N) \\
& +\frac{k-1+\alpha}{(2+\alpha) N-1} N_{k-1}(N)+\delta_{k, 1} .
\end{aligned}
$$

For $N \gg 1$, the recursion relation for the stationary degree distribution $n_{k}=N_{k}(N) / N$ is

$$
(k+2 \alpha+2) n_{k}=(k+\alpha-1) n_{k-1}+(2+\alpha) \delta_{k, 1} .
$$

Introducing the generating function $G(x)=\sum_{k=1}^{\infty} n_{k} x^{k}, G(x)$ is given as

$$
(2 \alpha+2-\alpha x) G(x)=\left(x^{2}-x\right) \frac{d G(x)}{d x}+(2+\alpha) x .
$$

One can easily calculate it to obtain

$$
G(x)=(\alpha+2) \frac{(1-x)^{\alpha+2}}{x^{2 \alpha+2}} \int_{0}^{x} d u \frac{u^{2 \alpha+2}}{(1-u)^{\alpha+3}} .
$$

Setting $1-x \equiv y, 1-u \equiv v$, and $v \equiv y t$, we obtain

$$
F(y) \equiv G(1-y)=\frac{2+\alpha}{(1-y)^{2 \alpha+2}} \int_{1}^{1 / y} d t \frac{(1-y t)^{2 \alpha+2}}{t^{\alpha+3}} .
$$

Here $\lim _{y \rightarrow 0^{+}} F(y)=\sum_{k=1}^{\infty} n_{k}, \quad \lim _{y \rightarrow 0^{+}} F^{\prime}(y)=-\sum_{k=1}^{\infty} k n_{k}, \quad$ and $\lim _{y \rightarrow 0^{+}} F^{\prime \prime}(y)=\sum_{k=1}^{\infty} k(k-1) n_{k}$, so we expand $F(y)$ up to the second term in $y$,

$$
\begin{aligned}
F(y) \simeq & (2+\alpha)[1+2(\alpha+1) y \\
& \left.+(\alpha+1)(2 \alpha+3) y^{2}\right] \int_{1}^{1 / y} \frac{d t}{t^{\alpha+3}}[1-2(\alpha+1) y t \\
& \left.+(\alpha+1)(2 \alpha+1) y^{2} t^{2}\right] \\
\simeq & (2+\alpha)(1+2(\alpha+1) y \\
& \left.+(\alpha+1)(2 \alpha+3) y^{2}\right)\left(\frac{1}{\alpha+2}-2 y\right. \\
& \left.+\frac{(\alpha+1)(2 \alpha+1)}{\alpha} y^{2}\right) .
\end{aligned}
$$

As a result, we obtain $\left.F(y)\right|_{y=0}=1,\left.\quad F^{\prime}(y)\right|_{y=0}=-2$, and $\left.F^{\prime \prime}(y)\right|_{y=0}=4(\alpha+1) / \alpha$, so we obtain an excess degree $k_{\text {ex }}$ of the GR tree with offset $\alpha$ as

$$
k_{\mathrm{ex}}=\frac{\left\langle k^{2}\right\rangle-\langle k\rangle}{\langle k\rangle}=2 \frac{\alpha+1}{\alpha} .
$$


[1] A.-L. Barabási and R. Albert, Science 286, 509 (1999).

[2] R. Albert and A.-L. Barabási, Rev. Mod. Phys. 74, 47 (2002).

[3] M. E. J. Newman, SIAM Rev. 45, 167 (2003).

[4] K. I. Goh, E. S. Oh, H. Jeong, B. Kahng, and D. Kim, Proc. Natl. Acad. Sci. U.S.A. 99, 12583 (2002).

[5] S. N. Dorogovtsev, A. V. Goltsev, and J. F. F. Mendes, Rev. Mod. Phys. 80, 1275 (2008).

[6] G. Bianconi, Phys. Lett. A 303, 166 (2002).

[7] M. Leone, A. Vázquez, A. Vespignani, and R. Zecchina, Eur. Phys. J. B 28, 191 (2002).

[8] F. Iglói and L. Turban, Phys. Rev. E 66, 036140 (2002).

[9] S. N. Dorogovtsev, A. V. Goltsev, and J. F. F. Mendes, Phys. Rev. E 66, 016104 (2002).

[10] K. Suchecki and J. A. Holyst, Phys. Rev. E 74, 011122 (2006).

[11] M. Hinczewski and A. N. Berker, Phys. Rev. E 73, 066126 (2006).

[12] M. Bauer, S. Coulomb, and S. N. Dorogovtsev, Phys. Rev. Lett. 94, 200602 (2005).

[13] A. Aleksiejuk, J. A. Holyst, and D. Stauffer, Physica A 310, 260 (2002).

[14] C. P. Herrero, Phys. Rev. E 69, 067109 (2004).

[15] H. Zhou and R. Lipowsky, Proc. Natl. Acad. Sci. U.S.A. 102, 10052 (2005).

[16] D. S. Callaway, J. E. Hopcroft, J. M. Kleinberg, M. E. J. Newman, and S. H. Strogatz, Phys. Rev. E 64, 041902 (2001); D. Lancaster, J. Phys. A 35, 1179 (2002); M. Coulomb and S. Bauer, Eur. Phys. J. B 35, 377 (2003).

[17] T. P. Eggarter, Phys. Rev. B 9, 2989 (1974).

[18] E. Müller-Hartmann and J. Zittartz, Phys. Rev. Lett. 33, 893 (1974).

[19] H. Matsuda, Prog. Theor. Phys. 51, 1053 (1974).
[20] J. von Heimburg and H. Thomas, J. Phys. C 7, 3433 (1974).

[21] H. Falk, Phys. Rev. B 12, 5184 (1975).

[22] R. Mélin, J. C. Anglès d'Auriac, P. Chandra, and B. Douçot, J. Phys. A 29, 5773 (1996).

[23] T. Stošić, B. D. Stošić, and I. P. Fittipaldi, J. Magn. Magn. Mater. 177-181, 185 (1998); B. D. Stošić, T. Stošić, and I. P. Fittipaldi, Physica A 355, 346 (2005).

[24] T. Hasegawa and K. Nemoto, Phys. Rev. E 75, 026105 (2007); T. Hasegawa and K. Nemoto, Physica A 387, 1404 (2008).

[25] S. N. Dorogovtsev, J. F. F. Mendes, and A. N. Samukhin, Phys. Rev. Lett. 85, 4633 (2000).

[26] P. L. Krapivsky, S. Redner, and F. Leyvraz, Phys. Rev. Lett. 85, 4629 (2000); P. L. Krapivsky and S. Redner, Phys. Rev. E 63, 066123 (2001); P. L. Krapivsky and S. Redner, J. Phys. A 35, 9517 (2002); P. L. Krapivsky and S. Redner, Phys. Rev. Lett. 89, 258703 (2002).

[27] G. Szabó, M. Alava, and J. Kertész, Phys. Rev. E 66, 026101 (2002).

[28] B. Bollobás and O. Riordan, Phys. Rev. E 69, 036114 (2004).

[29] A. Fekete, G. Vattay, and L. Kocarev, Phys. Rev. E 73, 046102 (2006).

[30] K. Malarz, J. Czaplicki, B. Kawecka-Magiera, and K. Kulakowski, Int. J. Mod. Phys. C 14, 1201 (2003); K. Malarz, J. Karpińska, A. Kardas, and K. Kulakowski, TASK Q. 8, 115 (2004); K. Malarz and K. Kulakowski, Eur. Phys. J. B 41, 333 (2004); K. Malarz and K. Kulakowski, Acta Phys. Pol. B 36, 2523 (2005).

[31] P. Bialas, Z. Burda, J. Jurkiewicz, and A. Krzywicki, Phys. Rev. E 67, 066106 (2003).

[32] Z. Burda, J. D. Correia, and A. Krzywicki, Phys. Rev. E 64, 046118 (2001). 